Louise A. Fletcher ${ }^{1}$, Yang Chen ${ }^{1}$, Paul Whitaker ${ }^{2}$, Miles Denton ${ }^{3}$, Daniel G. Peckham ${ }^{2}$ and Ian J. Clifton ${ }^{2}$ ${ }^{1}$ Dept of Civil Engineering, University of Leeds, Leeds, UK. ${ }^{2}$ Regional Adult Cystic Fibrosis Unit, St James's University Hospital, Leeds, UK. ${ }^{3}$ Dept of Microbiology, Leeds General Infirmary, Leeds, UK.

Correspondence: Ian J. Clifton, Regional Adult Cystic Fibrosis Unit, St James's University Hospital, Beckett Street, Leeds, LS9 7TF, UK. E-mail: i.clifton@nhs.net

Received: April 292016 | Accepted after revision: July 222016 | First published online: Sept 222016

Conflict of interest: None declared.

\title{
References
}

1 Floto RA, Olivier KN, Saiman L, et al. US Cystic Fibrosis Foundation and European Cystic Fibrosis Society consensus recommendations for the management of non-tuberculous mycobacteria in individuals with cystic fibrosis. Thorax 2016; 71: Suppl 1, i1-i22.

2 Esther CR Jr, Esserman DA, Gilligan P, et al. Chronic Mycobacterium abscessus infection and lung function decline in cystic fibrosis. J Cyst Fibros 2010; 9: 117-123.

3 Aitken ML, Limaye A, Pottinger P, et al. Respiratory outbreak of Mycobacterium abscessus subspecies massiliense in a lung transplant and cystic fibrosis center. Am J Respir Crit Care Med 2012; 185: 231-232.

4 Bryant JM, Grogono DM, Greaves D, et al. Whole-genome sequencing to identify transmission of Mycobacterium abscessus between patients with cystic fibrosis: a retrospective cohort study. Lancet 2013; 381: 1551-1560.

5 Bange FC, Brown BA, Smaczny C, et al. Lack of transmission of Mycobacterium abscessus among patients with cystic fibrosis attending a single clinic. Clin Infect Dis 2001; 32: 1648-1650.

6 O’Driscoll C, Konjek J, Heym B, et al. Molecular epidemiology of Mycobacterium abscessus complex isolates in Ireland. J Cyst Fibros 2016; 15: 179-185.

7 Harris KA, Underwood A, Kenna DT, et al. Whole-genome sequencing and epidemiological analysis do not provide evidence for cross-transmission of Mycobacterium abscessus in a cohort of pediatric cystic fibrosis patients. Clinl Infect Dis 2015; 60: 1007-1016.

8 Thomson R, Tolson C, Carter R, et al. Isolation of nontuberculous mycobacteria (NTM) from household water and shower aerosols in patients with pulmonary disease caused by NTM. J Clin Microbiol 2013; 51: 3006-3011.

9 Thomson R, Tolson C, Sidjabat H, et al. Mycobacterium abscessus isolated from municipal water: a potential source of human infection. BMC Infect Dis 2013; 13: 241.

10 Clifton IJ, Fletcher LA, Beggs CB, et al. A laminar flow model of aerosol survival of epidemic and non-epidemic strains of Pseudomonas aeruginosa isolated from people with cystic fibrosis. BMC Microbiol 2008; 8: 105.

11 Harris KA, Kenna DT, Blauwendraat C, et al. Molecular fingerprinting of Mycobacterium abscessus strains in a cohort of pediatric cystic fibrosis patients. J Clin Microbiol 2012; 50: 1758-1761.

12 Griffith DE, Aksamit T, Brown-Elliott BA, et al. An official ATS/IDSA statement: diagnosis, treatment, and prevention of nontuberculous mycobacterial diseases. Am J Respir Crit Care Med 2007; 175: 367-416.

13 Wainwright CE, France MW, O'Rourke P, et al. Cough-generated aerosols of Pseudomonas aeruginosa and other Gram-negative bacteria from patients with cystic fibrosis. Thorax 2009; 64: 926-931.

14 Clifton IJ, Fletcher LA, Beggs CB, et al. An aerobiological model of aerosol survival of different strains of Pseudomonas aeruginosa isolated from people with cystic fibrosis. J Cyst Fibros 2010; 9: 64-68.

\section{The impact of exposure to particulate air pollution from non-anthropogenic sources on hospital admissions due to pneumonia}

To the Editor:

Community-acquired pneumonia is a significant cause of morbidity and mortality among older adults [1]. The role of air pollution as a risk factor for pneumonia hospitalisations and mortality has been investigated [2,3] with most evidence coming from studies in North American and European cities, where anthropogenic sources are predominant in generating air pollution.

Particulate non-anthropogenic air pollution originating from dust is a common public health risk. Being located between the Sahara and the Arabian deserts (the world's largest dust-belt), the Negev region of Israel is exposed to extremely high levels of particulate matter originating from natural dust storms. During dust storms in this region, particulate matter levels can significantly exceed those defined as acceptable in terms of air quality and human health $\left(50 \mu \mathrm{g} \cdot \mathrm{m}^{-3}\right)$ with hourly concentrations of $100-5000 \mu \mathrm{g} \cdot \mathrm{m}^{-3}[4]$. Dust particles reach the southeastern Mediterranean by two main trajectories: one from the west (North 
Africa-Sinai-Negev) and the second from east (Arabian Desert-Negev) [5] with dust particles having somewhat different mineralogical and chemical compositions. In the winter, dust storms from the western sources are most prevalent, whereas dust storms from both west and east directions are frequent in the spring depending on the synoptic system [6]. Most of the intense storms with higher dust concentrations are associated with the western sources [4].

The unique combination of a centralised modern medical system and urban population residing in this arid and hot region makes the Negev an ideal "environmental laboratory" for studying the health effect of global environmental change such as desertification and global warming.

Previously, we have shown an association between dust exposure and risk of hospitalisation due to chronic obstructive pulmonary disease (COPD) [7] and asthma [8]. The aims of the present analyses were 1) to investigate the association between hospitalisations due to pneumonia and exposure to particles with a $50 \%$ cut-off aerodynamic diameter of $2.5 \mu \mathrm{m}$ (PM2.5) and $10 \mu \mathrm{m}$ (PM10) and 2) to identify individual characteristics that might modify the potential health effect.

Data from 4257 patients with 5611 hospitalisations admitted to Soroka University Medical Center (SUMC; Beersheba, Israel), a 1000-bed tertiary hospital between 2003-2013 due to pneumonia (ICD-9 codes 487, $486,481,480.8,514,482.41$ and 482.8) were included in this analysis. SUMC is the only hospital for a population of 730000 in Southern Israel. The following patient level data were obtained using the centralised electronic medical records database: diabetes (ICD-9 code 250), cardiovascular disease (ICD-9 codes 390-429), hypertension (ICD-9 code 401), COPD (ICD-9 codes 490, 491, 492 and 496) and socio-demographic data, such as sex and age.

Exposure assessment was based on a hybrid satellite-based spatio-temporally resolved model incorporating daily satellite-remote sensing data at a spatial resolution of $1 \times 1 \mathrm{~km}$ [9]. Briefly, we make use of a new algorithm developed by the US National Aeronautics and Space Administration (NASA): the Multi-Angle Implementation to Atmospheric Correction (MAIAC) [10], which provides aerosol optical depth (AOD) data at high resolutions. Using mixed-model frameworks, we regressed daily PM10 and PM2.5 mass concentration from the Ministry of Environmental Protection against AOD, temporal predictors (obtained from the Technion Center of Excellence in Exposure Science and Environmental Health air pollution monitoring database) and spatial predictors (obtained through the Israeli Central Bureau of Statistics and Survey Bureau mapping service). When AOD data were not available due to cloud coverage and non-retrieval days, we fitted a generalised additive model with a thin-plate spline term of latitude and longitude to interpolate the estimates. Good model performance was achieved, with out-of-sample cross validation R2 of 0.79 and 0.72 for PM10 and PM2.5, respectively. Both model predictions had little bias, with cross-validated slopes (predicted versus observed) of 0.99. More in depth details can be found in KLOOG et al. [9]. The daily average concentrations of the pollutants, estimated throughout the studied region, were assigned for each patient based on proximity to his geocoded home address. Daily data on air temperature and relative humidity for the study period were obtained from a central monitoring site located in the centre of the largest city of the region. We obtained daily meteorological data including temperatures $\left(\right.$ in ${ }^{\circ} \mathrm{C}$ ), and relative humidity from two monitoring stations located in the centre of the largest city (Be'er-Sheva) in the Negev area. PM10 and PM2.5 levels exceeded the World Health Organization-recommended daily guideline of 50 and $25 \mu \mathrm{g} \cdot \mathrm{m}^{-3}$ [11] in $36 \%$ and $17.1 \%$ of the study period days, respectively. The climate in the study region is relatively hot and dry, reaching a maximal daily average temperature of $31.4^{\circ} \mathrm{C}$ during the study period.

The data were analysed using the case-crossover approach. This association was estimated by odds ratios with $95 \%$ confidence intervals using conditional logistic regression model. Analyses were performed using R statistical software, version 3.0 (R Foundation for Statistical Computing, Vienna, Austria).

Using the novel exposure assessment we were able to show that natural particulate matter exposure increases the risk for hospital admission for pneumonia. The association between particulate matter was observed 5 days after the exposure (OR 1.051, 95\% CI 1.013-1.091, for increase in 10 units of PM2.5). The most susceptible subgroups to the PM2.5 and PM10 exposure were male patients older than 65 years (OR 1.108, 95\% CI 1.037-1.184, and OR 1.019, 95\% CI 1.000-1.040, respectively) or elderly patients with cardiovascular disease (OR 1.072, 95\% CI 1.023-1.123, and OR 1.016, 95\% CI 1.002-1.030, respectively) (table 1).

Desertification and global warming trends pose significant global ecological and environmental problems. Proximity of the Negev area to the Sahara and the Arabian deserts as important sources of mineral dust, highlights the importance of our findings and enhance our understanding of respiratory morbidity and its association with non-anthropogenic air pollution. Our finding with respect to association between exposure to particulate matter and risk of hospitalisation due to pneumonia are consistent with the results from a study from Ontario, Canada [2], and a study from the USA [3, 12]. Thus, our study strengthens the suggestion that air pollution may act as an irritant and induce defensive responses in airways, such as 
TABLE 1 Odds ratios and $95 \%$ confidence intervals for hospitalisation due to pneumonia associated with increase in 10 units of particulate matter

\begin{tabular}{|c|c|c|c|c|c|c|c|}
\hline \multirow[t]{2}{*}{ Pollutant } & \multirow[t]{2}{*}{ Mean $\pm s D$} & \multirow{2}{*}{$\begin{array}{l}\text { Interquartile } \\
\text { range }\end{array}$} & \multirow[t]{2}{*}{ Maximum } & \multicolumn{4}{|c|}{ OR $(95 \% \mathrm{CI})$} \\
\hline & & & & All $^{\#}$ & Age > 65 years & $\begin{array}{c}\text { Age }>65 \text { years } \\
+ \text { Male }\end{array}$ & $\begin{aligned} \text { Age } & >65 \text { years } \\
& + \text { CVD }\end{aligned}$ \\
\hline $\mathrm{PM} 10 \mu \mathrm{g} \cdot \mathrm{m}^{-3}$ & $54.31 \pm 43.8$ & 18.8 & 1531.9 & $1.007(0.996-1.019)$ & $1.018(1.005-1.031)$ & $1.019(1.000-1.040)$ & $1.016(1.002-1.030)$ \\
\hline $\mathrm{PM} 2.5 \mu \mathrm{g} \cdot \mathrm{m}^{-3}$ & $21.63 \pm 13.2$ & 6.2 & 471.6 & $1.051(1.013-1.091)$ & $1.078(1.032-1.127)$ & $1.108(1.037-1.184)$ & $1.072(1.023-1.123)$ \\
\hline
\end{tabular}

Odds ratios and $95 \%$ confidence intervals were estimated from conditional logistic regression analysis at its most statistically significant single lag (lag 5) adjusted for temperature and relative humidity. CVD: cardiovascular disease.": all sexes and ages.

increased mucus secretion and increased bronchial hyperreactivity [13]. In addition, particulate matter has been shown to produce free radicals and oxidative stress on lung cells. These reactions might lead to the tissue inflammation, resulting in exudative discharge to the alveoli. Radiologically, this will be evident as a consolidate, and the diagnosis of pneumonia can be established. Animal studies have shown an increased vulnerability to PM10 in animals with cardiopulmonary disease [14] and exacerbation of ongoing pneumococcal infection after exposure to concentrated ambient PM2.5 [15].

In conclusion, community-acquired pneumonia is a significant cause of morbidity and mortality among older adults. We found that short-term exposure to natural particulate matter increases the risk for hospital admission for pneumonia, particularly for older patients or patients with pre-existing cardiovascular disease.

@ERSpublications

Natural particulate matter linked to hospitalisation for pneumonia, particularly in older or cardiac patients http://ow.ly/ct4T303L4QP

Alina Vodonos ${ }^{1,2}$, Itai Kloog ${ }^{3}$, Liora Boehm ${ }^{4}$ and Victor Novack ${ }^{2}$

${ }^{1}$ Dept of Public Health, Faculty of Health Sciences, Ben-Gurion University of the Negev, Be'er-Sheva, Israel. ${ }^{2}$ Clinical Research Center, Soroka University Medical Center, Be'er-Sheva, Israel. ${ }^{3}$ Dept of Geography and Environmental Development, Faculty of Humanities and Social Sciences, Ben-Gurion University of the Negev, Be'er-Sheva, Israel. ${ }^{4}$ Soroka University Medical Center, Be'er-Sheva, Israel.

Correspondence: Victor Novack, Soroka Clinical Research Center, Soroka University Medical Center, Be'er-Sheva, Israel 84101. E-mail: victorno@clalit.org.il

Received: June 022016 | Accepted after revision: Aug 182016 | First published online: Nov 112016

Conflict of interest: None declared.

\section{References}

1 Marrie TJ. Community-acquired pneumonia in the elderly. Clin Infect Dis 2000; 31: 1066-1078.

2 Neupane B, Jerrett M, Burnett RT, et al. Long-term exposure to ambient air pollution and risk of hospitalization with community-acquired pneumonia in older adults. Am J Respir Crit Care Med 2010; 181: 47-53.

3 Zanobetti A, Schwartz J. Air pollution and emergency admissions in Boston, MA. J Epidemiol Community Health 2006; 60: 890-895.

4 Krasnov H, Katra I, Koutrakis P, et al. Contribution of dust storms to PM10 levels in an urban arid environment. J Air Waste Manage Assoc 2014; 64: 89-94.

5 Ganor E, Osetinsky I, Stupp A, et al. Increasing trend of African dust, over 49 years, in the eastern Mediterranean. $J$ Geophys Res 2010; 115: D07201.

6 Alpert P, Neeman B, Shay-El Y. Climatological analysis of Mediterranean cyclones using ECMWF data. Tellus A 1990; 42: 65-77.

7 Vodonos A, Friger M, Katra I, et al. The impact of desert dust exposures on hospitalizations due to exacerbation of chronic obstructive pulmonary disease. Air Qual Atmos Health 2014: 1-7.

8 Yitshak-Sade M, Novack V, Katra I, et al. Non-anthropogenic dust exposure and asthma medication purchase in children. Eur Respir J 2015; 45: 652-660.

9 Kloog I, Sorek-Hamer M, Lyapustin A, et al. Estimating daily PM 2.5 and PM 10 across the complex geo-climate region of Israel using MAIAC satellite-based AOD data. Atmos Environ 2015; 122: 409-416.

10 Lyapustin A, Wang Y, Laszlo I, et al. Multiangle implementation of atmospheric correction (MAIAC): 2. Aerosol algorithm. J Geophys Res Atmos 2011; 116.

11 World Health Organization. WHO Air Quality Guidelines for Particulate Matter, Ozone, Nitrogen Dioxide and Sulfur Dioxide. Global Update 2005, Summary of Risk Assessment. Geneva, WHO, 2006.

12 Medina-Ramon M, Zanobetti A, Schwartz J. The effect of ozone and PM10 on hospital admissions for pneumonia and chronic obstructive pulmonary disease: a national multicity study. Am J Epidemiol 2006; 163: 579-588.

13 Anderson HR, Atkinson RW, Bremner SA, et al. Particulate air pollution and hospital admissions for cardiorespiratory diseases: are the elderly at greater risk? Eur Respir J 2003; 40: Suppl. 39s-46s. 
14 Costa DL, Dreher KL. Bioavailable transition metals in particulate matter mediate cardiopulmonary injury in healthy and compromised animal models. Environ Health Perspect 1997; 105: Suppl. 5, 1053-1060.

15 Gurgueira SA, Lawrence J, Coull B, et al. Rapid increases in the steady-state concentration of reactive oxygen species in the lungs and heart after particulate air pollution inhalation. Environ Health Perspect 2002; 110: 749-755.

\section{Fatal acute respiratory distress syndrome with diffuse alveolar damage: donor lymphocyte infusion imputability?}

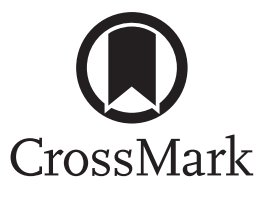

To the Editor:

Donor lymphocyte infusion (DLI) is increasingly used after allogeneic haematopoietic stem cell transplantation (allo-HSCT), as a pre-emptive or curative immunotherapy of relapsed haematological malignancies, enhancing graft-versus-tumour effects [1]. The main complication is graft-versus-host disease (GVHD), with cumulative incidences of $40-60 \%$ and 33-61\% for acute and chronic GVHD, respectively, typically developing 32-42 days after DLI [2]. Pulmonary complications after DLI are poorly documented. To our knowledge, there are no cases of diffuse alveolar damage (DAD) [3] after DLI in the literature to date. Here, we report our experience of two cases of acute respiratory distress syndrome (ARDS) with DAD following DLI after allo-HSCT, rapidly extending to fatal pulmonary fibrosis. Submission was approved by our institutional review board and informed consent was obtained.

A 68-year-old male patient (patient 1) with acute myeloid leukaemia received haplo-identical allo-HSCT after a thiotepa, fludarabine and busulfan conditioning regimen. No major complications occurred. Prophylactic haplo-DLI was performed at day 140. 4 days after, the patient developed fever and dyspnoea, without any symptoms of GVHD. Chest computed tomography (CT) revealed diffuse bilateral areas of ground-glass attenuation. The patient was admitted early to the intensive care unit (ICU) with isolated acute respiratory failure (ARF). Initial noninvasive infectious workup identified a co-infection with Klebsiella pneumoniae in sputa and human herpesvirus 6 (HHV6) in the nasopharyngeal aspirate. Repeated induced sputa for Pneumocystis jiroveci PCR were negative. All other infectious tests were negative. Cardiac echography was performed to rule out cardiac pulmonary oedema. We started antibiotics, ganciclovir and corticosteroids in light of the radiological appearance. The ventilation strategy consisted of high-flow oxygen and noninvasive ventilation (NIV). Respiratory failure progressed to ARDS [4]. Chest CT abnormalities worsened with extensive ground-glass opacities, traction bronchiectasis and subpleural honeycombing. We initiated empirical voriconazole, stopped it when negative $\beta$-D-glucan result was available and increased corticosteroids to $2 \mathrm{mg} \cdot \mathrm{kg}^{-1}$ per day. Because of the absence of improvement, CT-guided lung biopsy was performed. Histology revealed interstitial fibrosis with associated features compatible with DAD in the organising phase. Tumoural infiltration, GVHD, pulmonary veno-occlusive disease, granuloma and pathogens were excluded in the limit of the small biopsy (figure 1). The patient's respiratory status worsened, as did the chest CT scan fibrosis features, and the patient died after a 35-day ICU stay.

A 68-year-old male patient (patient 2) with myeloma underwent human leukocyte antigen-identical sibling allo-HSCT after a conditioning regimen consisting of fludarabine, thymoglobulin and busulfan. The patient was included in a phase I protocol of infusion of selected donor natural killer (NK) cells, currently under investigation (www.clinicaltrials.gov identifier NCT01853358). NK DLI was performed at day 103 post-allo-HSCT. 10 days later, he developed fever and dyspnoea. He was admitted to the ICU 30 days after DLI, with isolated ARF requiring $10 \mathrm{~L} \cdot \mathrm{min}^{-1}$ oxygen and no symptoms of GVHD. Chest CT revealed diffuse, bilateral, patchy ground-glass opacities with consolidation, traction bronchiectasis and a crazy-paving aspect. Noninvasive infectious work-up identified a co-infection with K. pneumoniae and adenovirus in respiratory samples. Cardiac pulmonary oedema was ruled out. Antibiotics, cidofovir and corticosteroids were initiated. The ventilation strategy consisted of high-flow oxygen and NIV. The patient's respiratory status worsened with ARDS development, requiring mechanical ventilation, prone position, neuromuscular blockade and nitric oxide. Microbiological samples were repeated, identifying Candida tropicalis, Candida albicans and Enterococcus faecium. P. jiroveci PCR was negative. In this context, as we could not distinguish 